\title{
Experiência da residência de medicina de família e comunidade no teleatendimento pelo monitora Covid-19
}

\author{
Experience report of family and community \\ medicine postgraduate on remote \\ assessment in monitora Covid-19
}

\section{Marcus Vinicius Sacramento França $\mathbb{C}$}

Escola Bahiana de Medicina e Saúde Pública (Salvador). Bahia, Brasil. marcus90franca@gmail.com

RESUMO | INTRODUÇÃO: A infecção pelo vírus SARS-CoV-2 causa a COVID-19, caracterizado por sintomas gripais como tosse, febre, odinofagia, alterações gustativas e olfativas, podendo complicar com pneumonia, síndrome respiratória aguda grave, sepse e eventos trombóticos. Com a transmissão comunitária no Brasil, no final de março, vários serviços não essenciais foram fechados e procedimentos eletivos adiados. A legalização temporária da telemedicina mostrou-se conveniente para as circunstâncias da pandemia, permitindo telemonitoramento e teleconsultas, como ocorre no aplicativo Monitora COVID-19. OBJETIVO: Relatar as potencialidades desses aplicativos no desenvolvimento de habilidades dos residentes de medicina de família e comunidade no acompanhamento dos casos de síndrome gripal. RELATO DE EXPERIÊNCIA: Por meio do processo de trabalho nesse aplicativo, reforçaram-se princípios de integralidade, multidisciplinariedade e o método clínico centrado na pessoa, tão importantes para saúde da família. O acompanhamento dos pacientes de maneira integral requereu o conhecimento das subjetividades, da experiência da doença e algumas vezes criação de vínculo. O aperfeiçoamento da habilidade comunicativa e competência cultural foram importantíssimos para boa relação e diálogo com o paciente no aplicativo. CONCLUSÃO: O aplicativo possibilitou a acompanhamento remoto de pacientes com síndrome gripal, orientando autocuidado e encaminhamento resolutivo nos casos graves. O papel da medicina de família e comunidade, seus princípios e práticas, mostram-se importantes para aperfeiçoamento das práticas de cuidado nessa ferramenta.

PALAVRAS-CHAVE: Medicina de família e comunidade. Residência médica. Infecção por coronavírus. Telemedicina.
ABSTRACT | INTRODUCTION: SARS-CoV-2 infection causes COVID-19, characterized by flu-like symptoms such as cough, fever, odynophagia, gustatory and olfactory changes, which may complicate pneumonia, severe acute respiratory syndrome, sepsis, and thrombotic events. Since March, community transmission in Brazil has been happening, then several non-essential services were closed and elective procedures postponed. The temporary legalization of telemedicine proved to be convenient for the COVID-19 pandemic, allowing telemonitoring and teleconsultations it occurs by Monitor COVID-19 application. OBJECTIVE: This article aims to report the potentialities of this application to develop skills in the postgraduate course in Family and Community Medicine in the follow-up of cases of COVID-19. EXPERIENCE REPORT: through the work process in this application, principles of integrality, multidisciplinarity, and the person-centered clinical method, so important for family health, were reinforced. The integral follow-up of patients required knowledge of subjectivities, disease, illness, and sometimes bonding. The improvement of communicative skills and cultural competence was very important for good relationships and dialogue with the patient in this application. CONCLUSION: this application allowed remote follow-up of patients with flu syndrome, guiding self-care and resolutive referral in severe cases. The role of family and community medicine, its principles, and practices are important for improving care practices in this tool.

Keywords: Family and community medicine. Medical residency. Infection from coronavirus. Telemedicine. 


\section{Introdução}

A infecção pelo vírus SARS-CoV-2 causa a COVID-19, doença identificada inicialmente na China, em dezembro de 2019, em quadros de pneumonia nos trabalhadores do mercado de Wuhan. Essa doença é caracterizada por sintomas gripais como tosse, febre, odinofagia, alterações gustativas e olfativas, podendo complicar com pneumonia, síndrome respiratória aguda grave, sepse e tromboses. Também se apresentar oligo ou assintomática. Em 30 de janeiro, a Organização Mundial de Saúde declarou o surto pelo novo coronavírus como uma emergência de Saúde Pública de importância internacional, e, em 11 de março de 2020, como uma pandemia. Segundo o Ministério da Saúde (2020), o primeiro caso identificado no Brasil deu-se em 26 de Fevereiro, em São Paulo, sendo identificados outros casos em Feira de Santana e Salvador no mês de março. ${ }^{1}$

O Estado brasileiro, em 20 de março de 2020, decretou status de transmissão comunitária em todo seu território, recomendando o fechamento de serviços não essenciais e postergação de atendimentos eletivos em saúde. Às unidades de saúde, designou-se como porta de entrada de casos leves e moderados; recomendou-se o diagnóstico sindrômico de gripe e o afastamento de todos os casos suspeitos por 14 dias a partir do início dos sintomas, devido ao potencial do quadro ter etiologia pelo coronavírus 2 e sua alta capacidade de transmissão.?

A legalização temporária da telemedicina nos moldes atuais possibilitou acompanhamento de pacientes à distância e redução de aglomerações em unidades de saúde, como abordam Santos et al.

"[...] a telemedicina e a telessaúde apresentam-se como instrumentos estratégicos, considerando-se as recentes resoluções do Conselho Federal de Medicina [...] as quais são favoráveis ao uso dessas ferramentas em caráter excepcional e temporário, diante do atual cenário de emergência de saúde pública" 3

Dentro desse contexto, o Monitora Covid-19 foi um aplicativo construído pela Secretaria de Ciência, Tecnologia e Inovação (SECTI), Secretaria de Saúde da Bahia (SESAB) e as empresas Novotech e Core para realizar o telemonitoramento de casos dessa síndrome.
Na Bahia, passou a ser utilizado em abril, com participação de residentes de programas de medicina de família e comunidade da Bahia, residências multiprofissionais e profissionais da Fundação Estatal de Saúde (FESF) em trabalho remoto. Nesse aplicativo, é possível realizar telemonitoramento, tele-orientação e encaminhamento de pacientes com suspeita do novo coronavírus, caso haja necessidade. Tal aplicativo permite a classificação dos usuários automaticamente, de acordo com patologias prévias, idade e sintomas referidos pelo paciente. ${ }^{4}$

Os atendimentos no aplicativo vêm sendo realizados por residentes de medicina de família e comunidade da Fundação Estatal de Saúde da Família (FESF), residentes em trabalho remoto da Escola Bahiana de Medicina e Saúde Pública (EBMSP), Escola de Saúde Pública da Bahia (ESPBA), profissionais da FESF em trabalho remoto, residentes multiprofissionais e outros profissionais. Há também parceira com internos da Universidade Federal da Bahia (UFBA) e profissionais da Universidade Federal do Sul da Bahia (UFSB). A participação de residentes de medicina de família em comunidade no aplicativo proporcionou uma experiência sobre os princípios e conhecimento dessa especialidade no contexto de teleatendimento e acompanhamento de pacientes com quadros sintomáticos agudos e residuais cronificados. Baseandose, quando possível, no método clínico centrado na pessoa. O objetivo desse artigo é relatar o processo de trabalho dos residentes em medicina de família e comunidade no aplicativo Monitora COVID-19, suas potencialidades e o desenvolvimento de habilidades específicas no acompanhamento de pacientes com síndrome gripal através dessa ferramenta.

\section{Relato de experiência}

De acordo com manual Orientações para atendimento clínico de retaguarda no Monitora COVID-19, organizado pelo Programa Integrado de Residências e a FESF-SUS, a classificação de risco dá-se automaticamente, de verde a vermelho, de acordo com o grau de gravidade e urgência de atendimento, no ato do cadastro para primeira consulta ou após cada atualização realizada pelo paciente. Os sintomas presentes no questionário respondido pelo usuário são: 
congestão nasal, mialgia, dispneia, desconforto torácico, palpitação, artralgia, náuseas, vômitos, diarreia, cefaleia, odinofagia, alterações no olfato e paladar. Esses pacientes adicionalmente são questionados quanto a presença de comorbidades. Em vigência de dispneia, dor torácica ou desconforto respiratório ou palpitação, os usuários são classificados como vermelhos - devendo serem atendidos prioritariamente por profissionais médicos, em até 24 horas. A presença de outros sintomas gripais em pacientes com comorbidades ou a partir de 60 anos define-os como risco laranja. Já a classificação amarela corresponde aos pacientes sem patologias prévias e abaixo de 60 anos com quadro suspeito. Pacientes verdes não possuem sinais e sintomas relacionados à COVID-19 ou estão assintomáticos, sendo orientados automaticamente pelo aplicativo. $\underline{5}$

Essa triagem inicial define a prioridade de atendimentos do dia, sendo os novos usuários vermelhos e laranjas, e reavaliações vermelhas atendidas prioritariamente por médicos. As reavaliações de pacientes laranjas e amarelos podem ser realizadas também pelos demais profissionais de saúde. Após atendimento, se necessário, reclassifica-se os usuários de acordo com sua impressão clínica, com base na história coletada e resultado de exames, informados pelo usuário. Além disso, os usuários são orientados sobre a atualização, em caso de novos sintomas. Em condições ideais de demanda, após primeiro atendimento, preconiza-se que os pacientes sejam reavaliados diariamente, priorizando os pacientes vermelhos novos e de seguimento, laranjas novos, e assim sucessivamente. O seguimento de usuários vermelhos, laranjas e amarelos deve ocorrer diariamente, porém em caso de grande volume de atendimentos no dia, acordou-se atender vermelhos em até 24 horas, laranjas em até 48 horas, e amarelos, em 72 horas. $\underline{5}$

Um ponto a ser ressaltado é a possibilidade de realizar atendimento via telefone ou acolhimento por mensagem, a fim de otimizar os atendimentos, principalmente durante momentos de demanda reprimida (reavaliação de laranjas ultrapassando 48 horas). Para isso, considera-se sintomas como diarreia, vômitos, disgeusia, anosmia, febre - considerados sinais importantes - e outras condições, como idade maior que 65 e duas ou mais comorbidades. $\frac{5}{}$

Quadro 1. Sintomas definidores para atendimento por telefone em pacientes amarelos e laranjas

\begin{tabular}{|l|l|}
\hline Principais sinais de gravidade & Outras condições \\
\hline - $\quad$ Náusea ou vômito & $\begin{array}{l}\text { Idoso (>65 anos) } \\
\text { OU } \\
2 \text { ou mais comorbidades/condições de saúde: } \\
\text { Diarreia } \\
\begin{array}{l}\text { Perda de olfato ou paladar } \\
\text { Febre }\end{array}\end{array}$ \\
& $\begin{array}{l}\text { hipertensão, diabetes, pacientes imunossuprimidos, } \\
\text { tabagista, asma, DPOC, Doença Renais, Hepáticas, }\end{array}$ \\
\hline
\end{tabular}

Fonte: FESF SUS, 2020.

Seguindo protocolo (Quadro 1), pacientes laranjas com dois ou mais dos sinais/sintomas associados a outras condições de saúde apresentam prioridade, em detrimento de outros pacientes com mesma classificação para atendimento por telefone. Já pacientes amarelos devem ser atendidos por ligação telefônica, caso apresentem 03 ou mais sintomas de gravidade. Em caso de não enquadramento nas condições relatadas, o paciente pode ser acolhido via mensagem, conforme padronização em manual Monitora COVID-19. Ainda assim, fica a critério do profissional definir pacientes com quadro não contemplado nos protocolos e que necessitem atendimento mais cuidadoso via telefone, conforme julgamento clínico. ${ }^{5}$

As avaliações dos profissionais são registradas no aplicativo, em formato de prontuário eletrônico, sendo possível também aos profissionais acompanhar a evolução do quadro e as últimas consultas. Após o teleatendimento, há uma ferramenta que permite o envio de mensagem ao paciente reforçando informações passadas por telefone. Isso possibilita ao usuário consultar as orientações referentes a precaução de contato, higiene, uso de máscara, alimentação balanceada e sinais de gravidade, sempre que houver necessidade. 
Trata-se de uma experiência nova no percurso profissional e formativo de vários residentes: o telemonitoramento e teleorientação de pacientes com suspeita de COVID-19. Inicialmente, tinha-se a impressão de que se tornaria apenas um serviço de triagem: pelo qual os pacientes mais graves seriam encaminhados a emergência. Contudo, isso foi apenas uma das potencialidades dessa ferramenta. O acompanhamento continuado desses pacientes, geralmente diário, possibilitou orientar melhor quanto a evolução da doença, os sintomas, o uso de medicações sintomáticas, terapias integrativas e complementares, autocuidado, orientação alimentar, distanciamento de familiares, uso de máscara, etiqueta respiratória, higienização de mãos, superfícies e ambientes, dentre outros. Essas orientações foram reforçadas ao longo das consultas e o processo de autocuidado foi (re) construído numa parceria profissional-usuário.

Além disso, possibilitou aos profissionais a aplicação do conteúdo teórico acerca da doença na práti$\mathrm{ca}$, dentro do contexto de adoecimento, respeitando subjetividades de cada paciente. Muitos usuários relatavam recuperação mais lenta do que o previsto em manuais e alguns, sobreposição de patologias como asma/COVID-19, transtornos de ansiedade/ síndrome gripal, arboviroses/ novo coronavírus, dentre outras associações. Tais hipóteses diagnósticas eram elencadas de acordo com relatos de sintomas, atendimentos prévios e escuta atenta. Sendo assim, a associação de conhecimentos de medicina baseada em evidências e o método clínico centrado na pessoa possibilitou aos residentes a ampliação o raciocínio clínico e o estabelecimento de novas formas de abordagem da pessoa adoecida, por meio de tecnologias de informação e comunicação.

Outro ponto importante que vêm sendo trabalhado é o estabelecimento de vínculo, tão importante para o médico de família e comunidade. Incrivelmente, foi possível estabelecer vínculo, mesmo à distância, e muitos pacientes depositaram confiança no profissional, de tal forma, que seguiam a conduta recomendada por especialistas focais, somente após segunda opinião do profissional do Monitora COVID-19. Isso remete a um dos princípios da Atenção Primária à Saúde, a coordenação do cuidado: o acompanhamento compartilhado do paciente com o especialista focal, porém com a integração do tratamento e cuidado continuado pelo médico da família e comunidade e equipe de saúde da família.
O método clínico centrado na pessoa ${ }^{6}$ mostrou-se indispensável no acompanhamento das pessoas, usuários do serviço. Esse método é constituído por quatro componentes que se inter-relacionam. $\mathrm{O}$ primeiro componente refere-se à concepção da saúde, doença e sua experiência. Aqui além dos componentes usuais do atendimento como anamnese e exames complementares, pode-se lançar mão dos sentimentos, expectativas em relação à doença e a funcionalidade do indivíduo no dia a dia. O segundo componente diz respeito a abordagem integral da pessoa, levando em consideração suas relações no contexto próximo (família, por exemplo) e no amplo (comunidade). Em seguida, a elaboração de um plano dá-se de forma conjunta, estabelecendo-se as responsabilidades da pessoa e do médico, além das prioridades terapêuticas. A intensificação da relação ocorre ao longo do tempo, estabelecendo-se o vínculo. ${ }^{\underline{7}}$

No atendimento pelo aplicativo, foram identificados vários tipos de pacientes: pessoas com sensação de adoecimento sem diagnóstico de patologia, pessoas com doença e adoecidas e pessoas com diagnóstico, mantendo sintomas leves e com capacidade de realizar suas atividades diárias, apesar de restritas devido ao isolamento social. Portanto, a experiência do adoecimento pelos indivíduos foi importante para pactuações quanto aos planos terapêuticos, diagnósticos e educativos. O segundo componente da medicina centrada na pessoa também pôde ser abordado durante os teleatendimentos: entendendo o individuo como um todo. Alguns pacientes com sintomas gripais não estavam realizando isolamento por serem provedores da casa. Nesse caso, o processo de convencimento através da educação em saúde auxiliou na redução dos riscos, porém nem sempre bem sucedido, visto a situação de vulnerabilidade social de muitas famílias e a falta de um Estado garantidor de direitos sociais básicos.

Durante as orientações, a importância da abordagem integral do sujeito para elaboração de um plano conjunto (componentes do método centrado na pessoa) foi premente. A avaliação do ambiente domiciliar, da quantidade de indivíduos residentes no domicílio, se sintomáticos ou não, condições socioeconômicas e modalidade de trabalho do paciente e contactantes foram importantes para elaboração do plano terapêutico e educacional. Em casas mais amplas, a possibilidade de realização de isolamento foi mais factível. Já em famílias grandes, residentes de casa com poucos cômodos, havia uma tentativa de 
adaptação para o distanciamento dentro das circunstâncias familiares e ambientais do sujeito. O quarto componente - intensificação da relação entre o médico e a pessoa - foi possível em casos mais arrastados, nos quais o paciente apresentava sintomas residuais ou comorbidades que dificultavam a regressão dos sintomas. Esse prolongamento do acompanhamento também ocorreu em casos de pacientes ansiosos e depressivos e/ou que apresentavam algum grau de somatização.

O paciente com sintomas gripais era avaliado por meio de várias perspectivas, não se restringindo aos aspectos médicos. Muitos pacientes com sintomas persistentes de COVID-19 ou com relatos de quadros recorrentes em pequeno intervalo de tempo apresentavam transtornos mentais de base ou questões familiares ou no trabalho. Sendo, talvez o sintoma não associado ao novo coronavírus, mas sim a repercussões físicas de demandas mentais não elaboradas. Nesses casos, foi importante atuação multidisciplinar junto a equipe Bem Estar, a qual dava apoio psicológico mais específico e qualificado, no campo da saúde mental.

Com o teleatendimento, foi possível desenvolver a capacidade de uma escuta sensível e qualificada, importantíssima para os profissionais de saúde, em especial na estratégia saúde da família. Pôde-se identificar, em alguns momentos, pessoas com desconforto respiratório através da fala, pacientes apreensivos, ansiosos, hipotímicos, dentre outros casos. Muitos pacientes em isolamento domiciliar sentiam-se solitários e usavam o momento da consulta para conversar e conectar-se com o mundo exterior, sendo a única oportunidade de interação com outro indivíduo, mesmo que por telefone. Portanto, algumas vezes as conversas não eram única e exclusivamente sobre a doença, mas também sobre a vida, sobre os sentimentos, medos, família, isolamento e o que o paciente trouxesse como demanda, impondo-se como processo terapêutico. Isso reforça um princípio da medicina de família e comunidade: o médico é um recurso da comunidade; sendo assim, esse deve acolher paciente com qualquer queixa, mesmo não sendo sua especialidade. O simples ato de falar e ser ouvido conferiu melhora de sintomas a muitos pacientes atendidos, reforçando a necessidade do diálogo, da escuta sensível e o potencial de cura através da palavra.

As sessões virtuais de aprendizagem constituíram ambientes pedagógicos, nos quais havia reunião de residentes multiprofissional em saúde da família e de medicina da família e comunidade, com discussão de casos acompanhados pelo aplicativo e de temas relevantes na área. Essa abordagem multidisciplinar possibilitou ampliar horizontes, aperfeiçoar abordagens como técnicas de relaxamento, orientações alimentares, autocuidado, orientações sobre atividade física no isolamento, além de aprofundamento teórico em temas como alterações sensoriais no COVID-19, telemonitoramento, saúde mental, aperfeiçoamento da escuta, reinfeção, dentre outros.

A Rede de Apoio ao Bem Estar constitui-se em um grupo interdisciplinar de profissionais capacitados para atender pacientes com algum tipo de sofrimento psíquico devido à pandemia ou prévio que viesse a piorar seus sintomas gripais. Para essa rede, apoiada pela Universidade Federal do Sul da Bahia, os profissionais do Monitora COVID encaminhavam pacientes com sintomas ansiosos, depressivos e psicossomáticos, segundo protocolos do manual para profissionais da retaguarda, a seguir ${ }^{5}$ :

"1) Você tem se sentido mais nervoso, irritado ou com muita tristeza? Nunca, às vezes ou sempre? 2) Aumentou o consumo de comida, bebida, cigarro ou outras substâncias? Nunca, às vezes ou sempre? 3) Você tem sentido aperto no peito, coração acelerado, vontade de chorar mais constante? Nunca, às vezes ou sempre? 4) Você perdeu algum familiar ou algum conhecido (próximo) recentemente? Sim ou não. 5) Você utiliza algum medicamento controlado? Nunca, às vezes ou sempre?" 5

Pessoas com, no mínimo, uma resposta sempre, duas às vezes ou morte de um familiar eram consideradas elegíveis ao encaminhamento para a rede de saúde mental, em caso de consentimento. Tal retaguarda mostrou-se importante no apoio a saúde mental de pacientes com síndrome gripal em um momento de extrema incerteza, solidão, isolamento, contribuindo para amenizar os sintomas e confortá-los. 
As habilidades de educação em saúde foram desenvolvidas durante a atuação no aplicativo, visto que o profissional lidou com diferentes indivíduos, com diferentes níveis socioeconômicos, escolaridades, religiões e cultura. No aplicativo, haviam usuários desde profissionais de saúde até pessoas totalmente leigas sobre o assunto. Portanto, aperfeiçoamento da habilidade comunicativa e competência cultural foram importantíssimos para boa relação e diálogo com o paciente. Mostrou-se importante também buscar compreender as impressões do paciente sobre a doença (experiência da doença), suas expectativas, suas condutas, autocuidado e cuidado com o próximo. A partir daí, construía-se um projeto terapêutico singular para aquele paciente, em parceria com o mesmo, através de um processo dialógico, empático e muitas vezes, emancipatório, como reforça Freire. ${ }^{?}$

Esse processo dialógico e dialético parte do pressuposto de que não há conhecimentos mais ou menos importantes e sim experiências e concepções diferentes. Sendo assim, torna-se necessário escutar os usuários a fim de construir planos colaborativos e coletivos, com maiores chances de sucesso, além de dialogar sobre as medidas de prevenção de contágio e tratamento adequado específico para cada caso, desmistificando algumas informações falsamente veiculadas por alguns representantes do Estado, como o uso da cloroquina. Estabelecia-se, via tecnologias de informação e comunicação, o encontro entre dois sujeitos subjetivos: o profissional de saúde e a pessoa. Cada um com suas subjetividades e seus conhecimentos construídos histórico-culturalmente, estando o profissional de saúde, na maioria das vezes, mais apropriado do conhecimento científico. Porém não mais importante que o conhecimento e as experiências do paciente. Essa relação horizontal e empática entre os sujeitos envolvidos possibilitou o diálogo e maiores chances de sucesso no telemonitoramento, além de confiança e respeito mútuo.

\section{Considerações finais}

O Monitora COVID-19 mostra-se como aplicativo importante para acompanhamento diário de pacientes com suspeita e/ou diagnóstico de COVID-19, evitando na maioria dos casos, que tais pacientes necessitem sair do isolamento, encaminhando-se a unidades de saúde para atendimento presencial, sem necessidade e reduzindo aglomerações, apresentando potencial coadjuvante no controle da transmissão do SARS-CoV-2.

O contato frequente entre profissionais e usuários permitiu estabelecer uma relação de confiança e vinculação à equipe do aplicativo, no momento da doença. Assim, foi possível sanar dúvidas, reduzir de apreensões sobre a evolução da doença, empoderar pacientes sobre seu protagonismo no processo de adoecimento, automonitoramento e cura. Tais ações juntamente com o estímulo a emancipação de sujeitos no processo de autocuidado e possibilitou encaminhamento responsável e resolutivo, em casos graves.

O trabalho interdisciplinar contribuiu para troca de experiências entre as diversas áreas e profissionais, atendimento integral aos usuários e atividades pedagógicas mais consistentes e compatíveis com um dos objetivos das residências em saúde da família: a abordagem multidisciplinar. As atividades docente-assistenciais com médicos, enfermeiros, odontólogos, fisioterapeutas, nutricionistas foram muito importantes para ampliar concepções acerca do tratamento e acompanhamento dos pacientes: abordagem da ansiedade, técnicas de relaxamento, orientação alimentar, dentre outros.

No tocante à residência de medicina de família e comunidade, a experiência neste aplicativo constituiu oportunidade única de aplicar princípios, conhecimentos tácitos, técnicas e habilidades indispensáveis para formação de especialistas em pessoas, tais como: método clínico centrado na pessoa, abordagem baseada em problemas, integralidade, habilidades de comunicação e escuta qualificada, raciocínio clínico, enfoque familiar e ambiental, educação em saúde, plano terapêutico adaptado a pessoa e seu contexto de adoecimento, competência cultural e estímulo a promoção e prevenção de agravos. 
Dentre os pontos negativos para residência, destacam-se redução da carga horária referente a disciplinas práticas presenciais envolvendo áreas importantes para formação do médico de família e comunidade, tais como atenção domiciliar, abordagem comunitária, territorialização, saúde da mulher, saúde da criança e pacientes com doenças crônicas. Além disso, o uso de tecnologias de informação e comunicação aumentaram a distância entre o médico e o paciente, o que se configura como uma dificuldade para garantir privacidade da consulta, o sigilo médico e o exame físico, primordiais para um fazer médico de excelência, em maioria dos casos.

Há necessidade de estudos para explorar e analisar as potencialidades dessa ferramenta de telemonitoramento para pacientes com outras condições clínicas, de maneira a otimizar o atendimento das equipes de saúde à população. A longo prazo, com a organização de redes de atenção à saúde e controle dos casos de COVID-19, será factível lançar mão da telemedicina para auxiliar às consultas convencionais. É possível que além de atividades de educação em saúde, casos leves e simples poderão ser resolvidos, possibilitando priorizando pacientes com maior vulnerabilidade e mais urgência para atendimento presencial. Bem como pacientes com doenças crônicas e com baixa adesão ao tratamento poderão ser monitorados com maior frequência sem necessidade de visitas domiciliares ou idas frequentes às unidades de saúde de família. Desse modo, cria-se uma atmosfera rica de possibilidades para otimizar a oferta de serviços assistenciais em saúde da família.

\section{Conflitos de interesses}

Nenhum conflito financeiro, legal ou político envolvendo terceiros (governo, empresas e fundações privadas, etc.) foi declarado para nenhum aspecto do trabalho submetido (incluindo, mas não se limitando a subvenções e financiamentos, participação em conselho consultivo, desenho de estudo, preparação de manuscrito, análise estatística, etc.).

\section{Referências}

1. Ministério da Saúde (Brasil), Secretaria de Atenção Primária à Saúde. Protocolo de Manejo Clínico do coronavírus na atenção primária [Internet]. Brasília, DF: Ministério da saúde; 2020. Disponível em: https://saude.rs.gov.br/upload/ arquivos/202004/14140606-4-ms-protocolomanejo-apsver07abril.pdf

2. Ministério da Saúde (Brasil), Secretaria de Vigilância em Saúde. Guia de Vigilância Epidemiológica: Emergência de Saúde Pública de Importância Nacional pela Doença pelo Coronavírus 2019 [Internet]. Brasília, DF: Ministérios da Saúde; 2020. Disponível em: https://portalarquivos.saude.gov.br/images/af gvs coronavirus_6ago20_ajustes-finais-2.pdf

3. Santos ABS, França MVS, Santos JLF. Atendimento remoto na APS no contexto da COVID-19: a experiência do Ambulatório da Comunidade da Escola Bahiana de Medicina e Saúde Pública em Salvador, Bahia. APS em Revista [Internet]. 2020;2(2):16976. Disponível em: https://apsemrevista.org/aps/article/ download/120/66/

4. FESF-SUS. Monitora COVID-19 [Internet]. Brasília: Fundação Estatal Saúde da Família. [citado em 2020 out 20]. Disponível em: http://www.fesfsus.ba.gov.br/covid19/monitora-covid-19/

5. Fundação Estatal Saúde da Família. Monitora COVID-19: Orientação para atendimento clínico de retaguarda. Salvador, BA: FESF-SUS, 2020.

6. Stewart M, Brown JB, McWhinney IR, McWilliam CL, Freeman TR. Medicina Centrada na Pessoa: transformando o método clínico. 3a. ed. Porto Alegre: Artmed; 2017.

7. Freire P. Pedagogia do oprimido. $50^{\mathrm{a}}$ ed. São Paulo: Paz e Terra; 2011. 